\title{
Stirling permutations, cycle structure of permutations and perfect matchings
}

\author{
Shi-Mei Ma* \\ School of Mathematics and Statistics \\ Northeastern University at Qinhuangdao \\ Hebei 066000, P.R. China \\ shimeimapapers@163.com
}

\author{
Yeong-Nan Yeh $^{\dagger}$ \\ Institute of Mathematics \\ Academia Sinica \\ Taipei, Taiwan \\ mayeh@math.sinica.edu.tw
}

Submitted: Jun 9, 2015; Accepted: Dec 10, 2015; Published: Dec 23, 2015

Mathematics Subject Classifications: 05A15, 05A19

\begin{abstract}
In this paper we provide constructive proofs that the following three statistics are equidistributed: the number of ascent plateaus of Stirling permutations of order $n$, a weighted variant of the number of excedances in permutations of length $n$ and the number of blocks with even maximal elements in perfect matchings of the set $\{1,2,3, \ldots, 2 n\}$.
\end{abstract}

Keywords: Stirling permutations; Excedances; Perfect matchings; Eulerian polynomials

\section{Introduction}

In this paper we will study the relationship between Stirling permutations, the cycle structure of permutations and perfect matchings, and will give constructive proofs for the equidistribution of some combinatorial statistics on these combinatorial structures.

Stirling permutations were introduced by Gessel and Stanley [6]. A Stirling permutation of order $n$ is a permutation of the multiset $\{1,1,2,2, \ldots, n, n\}$ such that every element between the two occurrences of $i$ is greater than $i$ for each $i \in[n]$, where $[n]=\{1,2, \ldots, n\}$. We refer the reader to $[1,7,8,11]$ for some recent results on Stirling permutations.

Let $\mathcal{Q}_{n}$ be the set of Stirling permutations of order $n$. For any $\sigma=\sigma_{1} \sigma_{2} \cdots \sigma_{2 n} \in \mathcal{Q}_{n}$, an occurrence of an ascent (resp. a plateau) is an index $i$ such that $\sigma_{i}<\sigma_{i+1}$ (resp. $\left.\sigma_{i}=\sigma_{i+1}\right)$. We say that an index $i \in[2 n-1]$ is an ascent plateau if $\sigma_{i-1}<\sigma_{i}=\sigma_{i+1}$,

\footnotetext{
*Supported by NSFC (11401083), the NSF of Hebei Province (A2013501070) and the Fundamental Research Funds for the Central Universities (N130423010).

†Supported by NSC 101-2115-M-001-013-MY3.
} 
where $\sigma_{0}=0$ (see [11]). Let ap $(\sigma)$ be the number of the ascent plateaus of $\sigma$. Then as an example, ap $(\mathbf{2 2 1 1 3 3 )}=2$. Let

$$
P_{n}(x)=\sum_{\sigma \in \mathcal{Q}_{n}} x^{\mathrm{ap}(\sigma)}=\sum_{k=1}^{n} P(n, k) x^{k} .
$$

Then $P_{n}(1)=\# \mathcal{Q}_{n}=(2 n-1) !$ !, where $(2 n-1) ! !$ is the double factorial of $2 n-1$. By [11, Theorem 3], the numbers $P(n, k)$ satisfy the recurrence relation

$$
P(n+1, k)=2 k P(n, k)+(2 n-2 k+3) P(n, k-1),
$$

with the initial values $P(1,1)=1$ and $P(1, k)=0$ for $k \leqslant 0$ or $k \geqslant 2$.

Let $\mathfrak{S}_{n}$ denote the symmetric group of all permutations of $[n]$ and $\pi=\pi_{1} \pi_{2} \cdots \pi_{n} \in$ $\mathfrak{S}_{n}$. An excedance in $\pi$ is an index $i$ such that $\pi_{i}>i$. Let exc $(\pi)$ denote the number of excedances in $\pi$. The classical Eulerian polynomials $A_{n}(x)$ are defined by

$$
A_{0}(x)=1, A_{n}(x)=\sum_{\pi \in \mathfrak{S}_{n}} x^{\mathrm{exc}(\pi)} \text { for } n \geqslant 1,
$$

and have been extensively investigated (see [4, 5] for instance). In [5], Foata and Schützenberger introduced a $q$-analog of the Eulerian polynomials defined by

$$
A_{n}(x ; q)=\sum_{\pi \in \mathfrak{S}_{n}} x^{\operatorname{exc}(\pi)} q^{\mathrm{cyc}(\pi)},
$$

where cyc $(\pi)$ is the number of cycles in $\pi$. Brenti $[2,3]$ further studied $q$-Eulerian polynomials and established the link with $q$-symmetric functions arising from plethysm. In particular, Brenti [3, Proposition 7.3] obtained the exponential generating function for $A_{n}(x ; q)$ :

$$
1+\sum_{n \geqslant 1} A_{n}(x ; q) \frac{z^{n}}{n !}=\left(\frac{1-x}{e^{z(x-1)}-x}\right)^{q} .
$$

For any $k \geqslant 1$, the $1 / k$-Eulerian polynomials $A_{n}^{(k)}(x)$ are defined by

$$
\sum_{n \geqslant 0} A_{n}^{(k)}(x) \frac{z^{n}}{n !}=\left(\frac{1-x}{e^{k z(x-1)}-x}\right)^{\frac{1}{k}} .
$$

Let $\mathbf{e}=\left(e_{1}, e_{2}, \ldots, e_{n}\right) \in \mathbb{Z}^{n}$. Let $I_{n, k}=\left\{\mathbf{e} \mid 0 \leqslant e_{i} \leqslant(i-1) k\right\}$, which is known as the set of $n$-dimensional $k$-inversion sequences. The number of ascents of $\mathbf{e}$ is defined by

$$
\operatorname{asc}(\mathbf{e})=\#\left\{i: 1 \leqslant i \leqslant n-1 \mid \frac{e_{i}}{(i-1) k+1}<\frac{e_{i+1}}{i k+1}\right\} .
$$

Recently, Savage and Viswanathan [12] discovered that

$$
A_{n}^{(k)}(x)=\sum_{\mathbf{e} \in I_{n, k}} x^{\mathrm{asc}(\mathbf{e})}=k^{n} A_{n}(x ; 1 / k) .
$$


A perfect matching of $[2 n]$ is a set partition of $[2 n]$ with blocks (disjoint nonempty subsets) of size exactly 2 . Let $\mathcal{M}_{2 n}$ be the set of matchings of [2n], and let $\mathrm{M} \in \mathcal{M}_{2 n}$. The standard form of $\mathrm{M}$ is a list of blocks $\left\{\left(i_{1}, j_{1}\right),\left(i_{2}, j_{2}\right), \ldots,\left(i_{n}, j_{n}\right)\right\}$ such that $i_{r}<j_{r}$ for all $1 \leqslant r \leqslant n$ and $1=i_{1}<i_{2}<\cdots<i_{n}$. Throughout this paper we always write $\mathrm{M}$ in standard form. It is well known that $\mathrm{M}$ can be regarded as a fixed-point-free involution on $[2 n]$. We call $(a, b)$ a marked block (resp. an unmarked block) if $b$ is even (resp. odd) and large than $a$. Let $\operatorname{mark}(\mathrm{M})$ be the number of marked blocks of $\mathrm{M}$.

Let

$$
N_{n}(x)=\sum_{\mathrm{M} \in \mathcal{M}_{2 n}} x^{\operatorname{mark}(\mathrm{M})}=\sum_{k=1}^{n} N(n, k) x^{k} .
$$

Then $N_{n}(1)=\# \mathcal{M}_{2 n}=(2 n-1)$ !!. The first few terms of $N_{n}(x)$ are

$$
N_{1}(x)=x, N_{2}(x)=2 x+x^{2}, N_{3}(x)=4 x+10 x^{2}+x^{3}, N_{4}(x)=8 x+60 x^{2}+36 x^{3}+x^{4} .
$$

The main purpose of the paper is to prove constructively that the following three statistics are equidistributed:

$\left(m_{1}\right)$ ap $(\sigma)$, the number of ascent plateaus of $\sigma \in \mathcal{Q}_{n}$;

$\left(m_{2}\right)$ a weighted variant of $\operatorname{exc}(\pi)$, where the variant is $n-\operatorname{exc}(\pi)$ and the weight is $2^{n-\operatorname{cyc}(\pi)}$

$\left(m_{3}\right) \operatorname{mark}(\mathrm{M})$, the number of marked blocks of $\mathrm{M} \in \mathcal{M}_{2 n}$.

The paper is organized as follows. In Section 2, we first derive the recurrence relation of the numbers $N(n, k)$ and then we give the connection between the three combinatorial objects studied in this paper on the level of generating functions. In Section 3, we give constructive proofs of the main results by using the $\mathcal{S P \mathcal { M }} \mathcal{M}$-sequences.

\section{Recurrence formula for $N(n, k)$}

Proposition 1. The numbers $N(n, k)$ satisfy the recurrence relation

$$
N(n+1, k)=2 k N(n, k)+(2 n-2 k+3) N(n, k-1),
$$

for $n, k \geqslant 1$, where $N(1,1)=1$ and $N(1, k)=0$ for $k \leqslant 0$ or $k \geqslant 2$.

Proof. For each $\mathrm{M} \in \mathcal{M}_{2 n}$ and for each block $(a, b)$ of $\mathrm{M}$, let $\varphi_{1}(\mathrm{M},(a, b)) \in \mathcal{M}_{2 n+2}$ be obtained from $\mathrm{M}$ by replacing the block $(a, b)$ with two blocks $(a, 2 n+1),(b, 2 n+2)$, and let $\varphi_{2}(\mathrm{M},(a, b)) \in \mathcal{M}_{2 n+2}$ be obtained from $\mathrm{M}$ by replacing the block $(a, b)$ with two blocks $(a, 2 n+2),(b, 2 n+1)$. Moreover, let $\varphi(\mathrm{M}) \in \mathcal{M}_{2 n+2}$ be obtained from M by adding the block $(2 n+1,2 n+2)$. It is obvious that for any $\mathrm{M}^{\prime} \in \mathcal{M}_{2 n+2}$, there is an $\mathrm{M} \in \mathcal{M}_{2 n}$ such that either $\mathrm{M}^{\prime}=\varphi(\mathrm{M})$ or there is a block $(a, b)$ of $\mathrm{M}$ such that $\mathrm{M}^{\prime}=\varphi_{1}(\mathrm{M},(a, b))$ or $\mathrm{M}^{\prime}=\varphi_{2}(\mathrm{M},(a, b))$. Moreover, it follows from the definition that $\operatorname{mark}(\varphi(\mathrm{M}))=\operatorname{mark}(\mathrm{M})+1$ and 
$(i)$ if $(a, b)$ is a marked block, then $\operatorname{mark}\left(\varphi_{i}(\mathrm{M},(\mathrm{a}, \mathrm{b}))\right)=\operatorname{mark}(\mathrm{M})$ for $i \in\{1,2\}$;

(ii) if $(a, b)$ is an unmarked block, then $\operatorname{mark}\left(\varphi_{i}(\mathrm{M},(\mathrm{a}, \mathrm{b}))\right)=\operatorname{mark}(\mathrm{M})+1$ for $i \in\{1,2\}$.

Assume $\mathrm{M}^{\prime}=\varphi(\mathrm{M})$ or $\varphi_{i}(\mathrm{M},(a, b))(i=1,2)$. If $\operatorname{mark}\left(\mathrm{M}^{\prime}\right)=k$, then $\operatorname{mark}(\mathrm{M})=k$ or $\operatorname{mark}(\mathrm{M})=k-1$. Each matching $\mathrm{M} \in \mathcal{M}_{2 n}$ with $\operatorname{mark}(\mathrm{M})=k$ corresponds to $2 k$ matchings $\mathrm{M}^{\prime} \in \mathcal{M}_{2 n+2}$ with $\operatorname{mark}\left(\mathrm{M}^{\prime}\right)=k$, and each matching $\mathrm{M} \in \mathcal{M}_{2 n}$ with $\operatorname{mark}(\mathrm{M})=k-1$ corresponds to $2(n-(k-1))+1$ matchings $\mathrm{M}^{\prime} \in \mathcal{M}_{2 n+2}$ with mark $\left(\mathrm{M}^{\prime}\right)=$ $k$. So

$$
N(n+1, k)=2 k N(n, k)+(2(n-(k-1))+1) N(n, k-1) .
$$

This completes the proof of (4).

Comparing (1) with (4), we see that the numbers $P(n, k)$ satisfy the same recurrence relation and initial conditions as $N(n, k)$. Hence they agree. The bijection $(a, b) \rightarrow\left(b^{\prime}, a^{\prime}\right)$ on $\mathcal{M}_{2 n}$ defined by $a^{\prime}=2 n+1-a, b^{\prime}=2 n+1-b$ shows that $N(n, k)$ is also the number of perfect matchings of $[2 n]$ in which exactly $k$ blocks with odd minimal elements. Now it is well known that the exponential generating function for $N_{n}(x)$ is given by (see $\left.[9,11]\right)$ :

$$
N(x, z)=\sum_{n \geqslant 0} N_{n}(x) \frac{z^{n}}{n !}=\sqrt{\frac{1-x}{1-x e^{2 z(1-x)}}} .
$$

By (2) and (5),

$$
A_{n}^{(2)}(x)=x^{n} N_{n}\left(\frac{1}{x}\right) .
$$

Therefore, it follows from (3) and (6) that

$$
N_{n}(x)=\sum_{\pi \in \mathfrak{S}_{n}} 2^{n-\operatorname{cyc}(\pi)} x^{n-\operatorname{exc}(\pi)} .
$$

\section{The $\mathcal{S P \mathcal { M }}$-sequences}

Let $Y_{n}=\left(y_{1}, y_{2}, \ldots, y_{n}\right)$ be a sequence of integers of length $n$. For $1 \leqslant k \leqslant n$, let POS $\left(Y_{k}\right)$ (resp. NPOS $\left(Y_{k}\right)$ ) denote the set of positive (resp. non-positive) entries of $Y_{k}=\left(y_{1}, y_{2}, \ldots, y_{k}\right)$. We define

$$
\operatorname{pos}\left(Y_{k}\right)=\# \operatorname{POS}\left(Y_{k}\right), \operatorname{npos}\left(Y_{k}\right)=\# \operatorname{NPOS}\left(Y_{k}\right) .
$$

Let $P_{k}$ be the set $\{1,2,3, \ldots, 2 k\}$ and let $N_{k}$ be the set $\{0,-1,-2, \ldots,-2 k\}$. In particular, $P_{0}=\emptyset, P_{1}=\{1,2\}, N_{0}=\{0\}$ and $N_{1}=\{0,-1,-2\}$.

Definition 2. Let $Y_{n}=\left(y_{1}, y_{2}, \ldots, y_{n}\right)$ be a sequence of integers of length $n$. We call the sequence $Y_{n}$ an $\mathcal{S P M} \mathcal{M}$-sequence of length $n$ if $y_{1}=0$, and $y_{k+1} \in P_{\operatorname{npos}\left(Y_{k}\right)} \cup N_{\operatorname{pos}\left(Y_{k}\right)}$ for $k=1,2,3, \ldots, n-1$. 
For example, $(0,1,-1,2,0)$ is a $\mathcal{S P} \mathcal{M}$-sequence, while $(0,1,-1,-4,2)$ is not since $y_{4}=-4 \notin P_{2} \cup N_{1}$. Let $\mathcal{S P} \mathcal{M}_{n}$ denote the set of $\mathcal{S P \mathcal { M }}$-sequences of length $n$. Note that $\operatorname{npos}\left(Y_{n-1}\right)+\operatorname{pos}\left(Y_{n-1}\right)=n-1$. This implies that the set $P_{\mathrm{npos}\left(Y_{n-1}\right)} \cup N_{\text {pos }\left(Y_{n-1}\right)}$ contains $2 n-1$ elements. So there are $2 n-1$ choices for $y_{n}$ and hence

$$
\# \mathcal{S P} \mathcal{M}_{n}=(2 n-1) \# \mathcal{S P} \mathcal{M}_{n-1}=(2 n-1) ! !
$$

We now present the first main result of this paper.

Theorem 3. For any $n \geqslant 1$,

$$
\sum_{\mathrm{M} \in \mathcal{M}_{2 n}} x^{\operatorname{mark}(\mathrm{M})}=\sum_{Y_{n} \in \mathcal{S P} \mathcal{M}_{n}} x^{\mathrm{npos}\left(Y_{n}\right)}=\sum_{\sigma \in \mathcal{Q}_{n}} x^{\mathrm{ap}(\sigma)}
$$

Let neg $\left(Y_{n}\right)$ and zero $\left(Y_{n}\right)$ be the number of negative entries and 0's in $Y_{n}$, respectively. Then $\operatorname{npos}\left(Y_{n}\right)=\operatorname{neg}\left(Y_{n}\right)+$ zero $\left(Y_{n}\right)$. The second main result is the following.

Theorem 4. For any $n \geqslant 1$,

$$
\sum_{Y_{n} \in \mathcal{S P} \mathcal{M}_{n}} x^{\operatorname{neg}\left(Y_{n}\right)} y^{\operatorname{zero}\left(Y_{n}\right)}=\sum_{\pi \in \mathfrak{S}_{n}} 2^{n-\operatorname{cyc}(\pi)} x^{n-\operatorname{cyc}(\pi)-\operatorname{exc}(\pi)} y^{\operatorname{cyc}(\pi)} .
$$

In particular,

$$
\sum_{Y_{n} \in \mathcal{S P} \mathcal{M}_{n}} x^{\operatorname{npos}\left(Y_{n}\right)}=\sum_{\pi \in \mathfrak{S}_{n}} 2^{n-\operatorname{cyc}(\pi)} x^{n-\operatorname{exc}(\pi)} .
$$

In the following subsections, we will give constructive proofs for the main results.

\subsection{A bijection between $\mathcal{S P \mathcal { M }}$-sequences and Perfect matchings}

We define

$$
\begin{aligned}
\mathcal{S P} \mathcal{M}_{n, k} & =\left\{Y_{n} \in \mathcal{S P} \mathcal{M}_{n} \mid \operatorname{npos}\left(Y_{n}\right)=k\right\} \\
\mathcal{M}_{2 n, k} & =\left\{\mathrm{M} \in \mathcal{M}_{2 n} \mid \operatorname{mark}(\mathrm{M})=k\right\} .
\end{aligned}
$$

Now we start to construct a bijection, denoted by $\Phi_{1}$, between $\mathcal{S P} \mathcal{M}_{n, k}$ and $\mathcal{M}_{2 n, k}$. When $n=1$, we have $y_{1}=0$. Set $\Phi_{1}\left(Y_{1}\right)=(1,2)$. This gives a bijection between $\mathcal{S P} \mathcal{M}_{1,1}$ and $\mathcal{M}_{2,1}$. Let $n=m$. Suppose $\Phi_{1}$ is a bijection between $\mathcal{S P} \mathcal{M}_{m, k}$ and $\mathcal{M}_{2 m, k}$ for all $k$. Consider the case $n=m+1$. Let $Y_{m+1}=\left(y_{1}, y_{2}, \ldots, y_{m}, y_{m+1}\right) \in \mathcal{S P} \mathcal{M}_{m+1}$. Then $Y_{m}=\left(y_{1}, y_{2}, \ldots, y_{m}\right) \in \mathcal{S P} \mathcal{M}_{m, k}$ for some $k$. Assume $\Phi_{1}\left(Y_{m}\right) \in \mathcal{M}_{2 m, k}$. Consider the following five cases:

(i) If $y_{m+1}=2 i-1>0$, then let $\Phi_{1}\left(Y_{m+1}\right)$ be obtained from $\Phi_{1}\left(Y_{m}\right)$ by replacing the $i$ th marked block $(c, d)$ by two blocks $(c, 2 m+1),(d, 2 m+2)$. In this case, $(c, 2 m+1)$ is an unmarked block and $(d, 2 m+2)$ is a marked block. Hence, $\operatorname{mark}\left(\Phi_{1}\left(\mathrm{Y}_{\mathrm{m}+1}\right)\right)=$ $\operatorname{mark}\left(\Phi_{1}\left(\mathrm{Y}_{\mathrm{m}}\right)\right)=\mathrm{k}$; 
(ii) If $y_{m+1}=2 i>0$, then let $\Phi_{1}\left(Y_{m+1}\right)$ be obtained from $\Phi_{1}\left(Y_{m}\right)$ by replacing the $i$ th marked block $(c, d)$ by two blocks $(c, 2 m+2),(d, 2 m+1)$. Hence, $\operatorname{mark}\left(\Phi_{1}\left(\mathrm{Y}_{\mathrm{m}+1}\right)\right)=$ $\operatorname{mark}\left(\Phi_{1}\left(\mathrm{Y}_{\mathrm{m}}\right)\right)=\mathrm{k}$;

(iii) If $y_{m+1}=-(2 i-1)<0$, then let $\Phi_{1}\left(Y_{m+1}\right)$ be obtained from $\Phi_{1}\left(Y_{m}\right)$ by replacing the $i$ th unmarked block $(e, f)$ by two blocks $(e, 2 m+1),(f, 2 m+2)$. In this case, $\operatorname{mark}\left(\Phi_{1}\left(\mathrm{Y}_{\mathrm{m}+1}\right)\right)=\operatorname{mark}\left(\Phi_{1}\left(\mathrm{Y}_{\mathrm{m}}\right)\right)+1=\mathrm{k}+1$;

(iv) If $y_{m+1}=-2 i<0$, then let $\Phi_{1}\left(Y_{m+1}\right)$ be obtained from $\Phi_{1}\left(Y_{m}\right)$ by replacing the $i$ th unmarked block $(e, f)$ by two blocks $(e, 2 m+2),(f, 2 m+1)$. In this case, $\operatorname{mark}\left(\Phi_{1}\left(\mathrm{Y}_{\mathrm{m}+1}\right)\right)=\operatorname{mark}\left(\Phi_{1}\left(\mathrm{Y}_{\mathrm{m}}\right)\right)+1=\mathrm{k}+1$;

(v) If $y_{m+1}=0$, then let $\Phi_{1}\left(Y_{m+1}\right)$ be obtained from $\Phi_{1}\left(Y_{m}\right)$ by appending the marked block $(2 m+1,2 m+2)$ right after $\Phi_{1}\left(Y_{m}\right)$. In this case,

$$
\operatorname{mark}\left(\Phi_{1}\left(\mathrm{Y}_{\mathrm{m}+1}\right)\right)=\operatorname{mark}\left(\Phi_{1}\left(\mathrm{Y}_{\mathrm{m}}\right)\right)+1=\mathrm{k}+1
$$

After the above step, we write the obtained perfect matching $\Phi_{1}\left(Y_{m+1}\right)$ in standard form. It is easy to verify that if $y_{m+1} \in P_{\mathrm{npos}\left(Y_{m}\right)}$ (resp. $\left.y_{m+1} \in N_{\operatorname{pos}\left(Y_{m}\right)}\right)$, then $\Phi_{1}\left(Y_{m+1}\right) \in$ $\mathcal{M}_{2 m+2, k}$ (resp. $\left.\Phi_{1}\left(Y_{m+1}\right) \in \mathcal{M}_{2 m+2, k+1}\right)$. By induction, we see that $\Phi_{1}$ is the desired bijection between $\mathcal{S P} \mathcal{M}_{n, k}$ and $\mathcal{M}_{2 n, k}$, which also gives a constructive proof of the left equality of (7).

In the following example, if $(a, b)$ is a marked block, then we put the entries $a$ and $b$ into a square bracket, i.e., replace $(a, b)$ by $[a, b]$. Otherwise, the parenthesis that contains $a$ and $b$ unchanged.

Example 5. Let $Y_{4}=(0,1,-2,4)$. The correspondence between $Y_{4}$ and $\Phi_{1}\left(Y_{4}\right)$ is built up as follows:

$$
\begin{aligned}
0 & \Leftrightarrow\{[1,2]\} \\
1 & \rightarrow\{[1,2]\} \Leftrightarrow\{(1,3),[2,4]\} ; \\
-2 & \rightarrow\{(1,3),[2,4]\} \Leftrightarrow\{[1,6],(3,5),[2,4]\}=\{[1,6],[2,4],(3,5)\} ; \\
4 & \rightarrow\{[1,6],[2,4],(3,5)\} \Leftrightarrow\{[1,6],[2,8],(4,7),(3,5)\}=\{[1,6],[2,8],(3,5),(4,7)\} .
\end{aligned}
$$

\section{$3.2 \quad$ A bijection between $\mathcal{S P \mathcal { M }}$-sequences and Stirling permutations}

Let $\mathcal{Q}\left(P_{\text {npos }\left(Y_{k}\right)} \cup N_{\text {pos }\left(Y_{k}\right)}\right)$ be the set of Stirling permutations of order $k$ with exactly $\operatorname{npos}\left(Y_{k}\right)$ ascent plateaus. In particular, $\mathcal{Q}\left(P_{1} \cup N_{0}\right)=\{11\}, \mathcal{Q}\left(P_{1} \cup N_{1}\right)=\{2211,1221\}$ and $\mathcal{Q}\left(P_{2} \cup N_{0}\right)=\{1122\}$. We now introduce a definition of labeled Stirling permutations.

Definition 6. Let $\sigma \in \mathcal{Q}\left(P_{\mathrm{npos}\left(Y_{k}\right)} \cup N_{\operatorname{pos}\left(Y_{k}\right)}\right)$. If $i_{1}<i_{2}<\ldots<i_{\text {npos }\left(Y_{k}\right)}$ are the ascent plateaus of $\sigma$, then we put the superscript labels $2 \ell-1$ before $i_{\ell}$ and $2 \ell$ after it, where $1 \leqslant \ell \leqslant \operatorname{npos}\left(Y_{k}\right)$. In the remaining positions, we put the superscript labels $-1,-2, \ldots,-2 \operatorname{pos}\left(Y_{k}\right)$ and 0 from left to right. 
As an example, the labeled version of 13324421 is given by ${ }^{-1} 1^{1} 3^{2} 3^{-2} 2^{3} 4^{4} 4^{-3} 2^{-4} 1^{0}$. We define

$$
\mathcal{Q}_{n, k}=\left\{\sigma \in \mathcal{Q}_{n} \mid \operatorname{ap}(\sigma)=k\right\} .
$$

Now we start to construct a bijection, denoted by $\Phi_{2}$, between $\mathcal{S P} \mathcal{M}_{n, k}$ and $\mathcal{Q}_{n, k}$. When $n=1$, we have $y_{1}=0$. Set $\Phi_{2}\left(Y_{1}\right)=11$. This gives a bijection between $\mathcal{S P} \mathcal{M}_{1,1}$ and $\mathcal{Q}_{1,1}$. Let $n=m$. Suppose $\Phi_{2}$ is a bijection between $\mathcal{S P} \mathcal{M}_{m, k}$ and $\mathcal{Q}_{m, k}$ for all $k$. Consider the case $n=m+1$. Let $Y_{m+1}=\left(y_{1}, y_{2}, \ldots, y_{m}, y_{m+1}\right) \in \mathcal{S P} \mathcal{M}_{m+1}$. Then $Y_{m}=\left(y_{1}, y_{2}, \ldots, y_{m}\right) \in \mathcal{S P} \mathcal{M}_{m, k}$ for some $k$. Assume $\Phi_{2}\left(Y_{m}\right) \in \mathcal{Q}_{m, k}$. Consider the following three cases:

(i) If $y_{m+1}=i>0$, then let $\Phi_{2}\left(Y_{m+1}\right)$ be obtained from $\Phi_{2}\left(Y_{m}\right)$ by inserting the pair $(m+1)(m+1)$ to the position of $\Phi_{2}\left(Y_{m}\right)$ with label $i$. Hence $Y_{m+1} \in \mathcal{S P} \mathcal{M}_{m+1, k}$ and $\Phi_{2}\left(Y_{m+1}\right) \in \mathcal{Q}_{m+1, k}$.

(ii) If $y_{m+1}=-i<0$, then let $\Phi_{2}\left(Y_{m+1}\right)$ be obtained from $\Phi_{2}\left(Y_{m}\right)$ by inserting the pair $(m+1)(m+1)$ to the position of $\Phi_{2}\left(Y_{m}\right)$ with label $-i$. Hence $Y_{m+1} \in \mathcal{S P} \mathcal{M}_{m+1, k+1}$ and $\Phi_{2}\left(Y_{m+1}\right) \in \mathcal{Q}_{m+1, k+1}$.

(iii) If $y_{m+1}=0$, then let $\Phi_{2}\left(Y_{m+1}\right)$ be obtained from $\Phi_{2}\left(Y_{m}\right)$ by appending the pair $(m+1)(m+1)$ right after $\Phi_{2}\left(Y_{m}\right)$. Hence $Y_{m+1} \in \mathcal{S P} \mathcal{M}_{m+1, k+1}$ and $\Phi_{2}\left(Y_{m+1}\right) \in$ $\mathcal{Q}_{m+1, k+1}$.

By induction, we see that $\Phi_{2}$ is the desired bijection between $\mathcal{S P} \mathcal{M}_{n, k}$ and $\mathcal{Q}_{n, k}$ for all $k$, which also gives a constructive proof of the right equality of (7).

Example 7. Let $Y_{4}=(0,2,-1,3)$. The correspondence between $Y_{4}$ and $\Phi_{2}\left(Y_{4}\right)$ is built up as follows:

$$
\begin{aligned}
0 & \Leftrightarrow 11 \\
2 & \rightarrow^{1} 1^{2} 1^{0} \Leftrightarrow 1221 \\
-1 & \rightarrow^{-1} 1^{1} 2^{2} 2^{-2} 1^{0} \Leftrightarrow 331221 \\
3 & \rightarrow^{1} 3^{2} 3^{-1} 1^{3} 2^{4} 2^{-2} 1^{0} \Leftrightarrow 33144221
\end{aligned}
$$

\subsection{A map from $\mathcal{S P \mathcal { M }}$-sequences to permutations}

In the following discussion, we always write $\pi \in \mathfrak{S}_{n}$ by its standard cycle decomposition, in which each cycle is written with its smallest entry first and the cycles are written in ascending order of their smallest entry. We now introduce a definition of labeled permutations.

Definition 8. Let $\pi \in \mathfrak{S}_{n}$ with $p$ excedances. If $i_{1}<i_{2}<\cdots<i_{p}$ are the excedances, then we put superscript labels $-k$ between $i_{k}$ and $\pi\left(i_{k}\right)$, where $1 \leqslant k \leqslant p$. In the remaining positions except the first position of each cycle, we put the superscript labels $1,2, \ldots, n-p$ from left to right. 
The number of anti-excedances of $\pi \in \mathfrak{S}_{n}$ is defined by aexc $(\pi)=n-\operatorname{exc}(\pi)$. Let

$$
\mathfrak{S}_{n, k}=\left\{\pi \in \mathfrak{S}_{n} \mid \operatorname{aexc}(\pi)=k\right\} .
$$

Now we start to construct a map, denoted by $\Phi_{3}$, from $\mathcal{S P} \mathcal{M}_{n, k}$ to $\mathfrak{S}_{n, k}$. When $n=1$, we have $y_{1}=0$. Set $\Phi_{3}\left(Y_{1}\right)=(1)$. This gives a map from $\mathcal{S P} \mathcal{M}_{1,1}$ to $\mathfrak{S}_{1,1}$. Let $n=m$. Suppose $\Phi_{3}$ is a map from $\mathcal{S P \mathcal { P }} \mathcal{M}_{m, k}$ to $\mathfrak{S}_{m, k}$ for all $k$ and there are $2^{m-t}$ sequences in $\mathcal{S P} \mathcal{M}_{m, k}$ are mapped to one permutation $\pi \in \mathfrak{S}_{m, k}$ with cyc $(\pi)=t$. Consider the case $n=m+1$. Let $Y_{m+1}=\left(y_{1}, y_{2}, \ldots, y_{m}, y_{m+1}\right) \in \mathcal{S P} \mathcal{M}_{m+1}$. Then $Y_{m}=\left(y_{1}, y_{2}, \ldots, y_{m}\right) \in$ $\mathcal{S P} \mathcal{M}_{m, k}$ for some $k$. Assume $\Phi_{3}\left(Y_{m}\right) \in \mathfrak{S}_{m, k}$. Consider the following three cases:

(i) If $y_{m+1}=2 i-1>0$ or $y_{m+1}=2 i>0$, then let $\Phi_{3}\left(Y_{m+1}\right)$ be obtained from $\Phi_{3}\left(Y_{m}\right)$ by inserting the entry $m+1$ to the position of $\Phi_{3}\left(Y_{m}\right)$ with label $i$. Since $\operatorname{exc}\left(\Phi_{3}\left(Y_{m+1}\right)=\operatorname{exc}\left(\Phi_{3}\left(Y_{m}\right)+1=m-k+1\right.\right.$, we have aexc $\left(\Phi_{3}\left(Y_{m+1}\right)\right)=k$. Moreover, $\operatorname{cyc}\left(\Phi_{3}\left(Y_{m+1}\right)\right)=\operatorname{cyc}\left(\Phi_{3}\left(Y_{m}\right)\right)$. Therefore, we have $2 \cdot 2^{m-t}=2^{m+1-t}$ sequences in $\mathcal{S P} \mathcal{M}_{m+1, k}$ that are mapped to one permutation $\pi \in \mathfrak{S}_{m+1, k}$ with $\operatorname{cyc}(\pi)=t$.

(ii) If $y_{m+1}=-(2 i-1)<0$ or $y_{m+1}=-2 i<0$, then let $\Phi_{3}\left(Y_{m+1}\right)$ be obtained from $\Phi_{3}\left(Y_{m}\right)$ by inserting the entry $m+1$ to the position of $\Phi_{3}\left(Y_{m}\right)$ with label $-i$. Since $\operatorname{exc}\left(\Phi_{3}\left(Y_{m+1}\right)=\operatorname{exc}\left(\Phi_{3}\left(Y_{m}\right)=m-k\right.\right.$, we have aexc $\left(\Phi_{3}\left(Y_{m+1}\right)\right)=k+1$. Moreover, $\operatorname{cyc}\left(\Phi_{3}\left(Y_{m+1}\right)\right)=\operatorname{cyc}\left(\Phi_{3}\left(Y_{m}\right)\right)$. Therefore, we have $2 \cdot 2^{m-t}=2^{m+1-t}$ sequences in $\mathcal{S P} \mathcal{M}_{m+1, k+1}$ that are mapped to one permutation $\pi \in \mathfrak{S}_{m+1, k+1}$ with $\operatorname{cyc}(\pi)=t$.

(iii) If $y_{m+1}=0$, then let $\Phi_{3}\left(Y_{m+1}\right)$ be obtained from $\Phi_{3}\left(Y_{m}\right)$ by appending a new cycle $(m+1)$ right after $\Phi_{3}\left(Y_{m}\right)$. Since exc $\left(\Phi_{3}\left(Y_{m+1}\right)=\operatorname{exc}\left(\Phi_{3}\left(Y_{m}\right)=m-k\right.\right.$, we have aexc $\left(\Phi_{3}\left(Y_{m+1}\right)\right)=k+1$. Moreover, $\operatorname{cyc}\left(\Phi_{3}\left(Y_{m+1}\right)\right)=\operatorname{cyc}\left(\Phi_{3}\left(Y_{m}\right)\right)+1$. Therefore, we have $1 \cdot 2^{m-t}=2^{m-t}$ sequences in $\mathcal{S P} \mathcal{M}_{m+1, k+1}$ that are mapped to one permutation $\pi \in \mathfrak{S}_{m+1, k+1}$ with $\operatorname{cyc}(\pi)=t+1$.

After the above step, we first write the obtained $\Phi_{3}\left(Y_{m+1}\right)$ in standard cycle decomposition, and then label it. By induction, we see that $\Phi_{3}$ is the desired map from $\mathcal{S P} \mathcal{M}_{n, k}$ to $\mathfrak{S}_{n, k}$, which also gives a constructive proof of $(8)$.

Example 9. Given $\pi=\left(\begin{array}{llll}1 & 3 & 5 & 2\end{array}\right)$ (4). The map $\Phi_{3}$ can be done if you proceed as follows:

$$
\begin{aligned}
y_{1}=0 & \rightarrow\left(1^{1}\right) ; \\
y_{2}=1 \text { or } y_{2}=2 & \rightarrow\left(1^{-1} 2^{1}\right) ; \\
y_{3}=-1 \text { or } y_{3}=-2 & \rightarrow\left(1^{-1} 3^{1} 2^{2}\right) ; \\
y_{4}=0 & \rightarrow\left(1^{-1} 3^{1} 2^{2}\right)\left(4^{3}\right) ; \\
y_{5}=1 \text { or } y_{5}=2 & \rightarrow\left(1^{-1} 3^{-2} 5^{1} 2^{2}\right)\left(4^{3}\right) .
\end{aligned}
$$

Hence, by $\Phi_{3}$, there are eight $\mathcal{S P M} \mathcal{M}$-sequences that are mapped to $\pi$ :

$$
\begin{aligned}
& (0,1,-1,0,1) ;(0,1,-1,0,2) ;(0,1,-2,0,1) ;(0,1,-2,0,2) ; \\
& (0,2,-1,0,1) ;(0,2,-1,0,2) ;(0,2,-2,0,1) ;(0,2,-2,0,2) .
\end{aligned}
$$




\section{Concluding remarks}

The key observation of the paper is the recurrence relation (4), from which we discover the relationship among Stirling permutations, the cycle structure of permutations and perfect matchings. We believe that the techniques developed in the paper can be used to deal with various combinatorial structures. Moreover, it would be interesting to study pattern avoidance properties of the $\mathcal{S P \mathcal { M }} \mathcal{M}$-sequences.

\section{Acknowledgements}

The authors thank the referee for his valuable suggestions which lead to a substantial improvement of the paper. They also thank Chan-Liang Chung for his valuable discussion.

\section{References}

[1] M. Bóna, Real zeros and normal distribution for statistics on Stirling permutations defined by Gessel and Stanley, SIAM J. Discrete Math., 23:401-406, 2008/09.

[2] F. Brenti. q-Eulerian polynomials arising from Coxeter groups. European J. Combin., 15:417-441, 1994.

[3] F. Brenti. A class of $q$-symmetric functions arising from plethysm. J. Combin. Theory Ser. A, 91:137-170, 2000.

[4] K. Dilks, T.K. Petersen, J.R. Stembridge. Affine descents and the Steinberg torus. Adv. in Appl. Math., 42:423-444, 2009.

[5] D. Foata, M. Schützenberger. Théorie Géométrique des Polynômes Euleriens. Lecture Notes in Mathematics, vol. 138, Springer-Verlag, Berlin-New York, 1970.

[6] I. Gessel and R.P. Stanley. Stirling polynomials. J. Combin. Theory Ser. A, 24:25-33, 1978.

[7] J. Haglund, M. Visontai. Stable multivariate Eulerian polynomials and generalized Stirling permutations, European J. Combin., 33:477-487, 2012.

[8] S. Janson, M. Kuba and A. Panholzer. Generalized Stirling permutations, families of increasing trees and urn models, J. Combin. Theory Ser. A, 118:94-114, 2011.

[9] S.-M. Ma. A family of two-variable derivative polynomials for tangent and secant. Electron. J. Combin., 20(1): \#P11, 2013.

[10] S.-M. Ma. Some combinatorial arrays generated by context-free grammars. European J. Combin., 34:1081-1091, 2013.

[11] S.-M. Ma, T. Mansour. The $1 / k$-Eulerian polynomials and $k$-Stirling permutations. Discrete Math., 338:1468-1472, 2015.

[12] C.D. Savage and G. Viswanathan. The $1 / k$-Eulerian polynomials. Electron. J. Combin., 19(1): \#P9, 2012. 